\title{
Coefficients de dose après incorporation d'iode 129. Influence de l'apport alimentaire
}

\author{
J.L. MALARBET*, A. AURENGO**, M. ROY***, B. LE GUEN* \\ C. DEVILLERS***, H. MÉTIVIER***
}

(Manuscrit reçu le 18 juillet 1997, accepté le 6 octobre 1997)

\begin{abstract}
RÉSUMÉ Les coefficients de dose, pour la contamination interne publiés par la CIPR et repris dans la nouvelle directive européenne permettent de calculer directement la dose efficace engagée en multipliant la quantité de radionucléide incorporée, exprimée en becquerels, Bq, par ces coefficients. Pour l'iode, cependant, cette méthodologie doit être adaptée, car le transfert de l'iode du sang vers la thyroïde dépend de l'apport quotidien d'iode : plus cet apport est grand, moins la fraction prélevée par la glande sera grande. Dans le cas de l'isotope 129 radioactif de l'iode, par exemple, pour un même Bq ingéré le facteur de captage peut varier de 0,3 si l'apport quotidien est de $165 \mu \mathrm{g}$, à 0,03 si l'apport quotidien est de 2,2 mg. Pour illustrer ces variations, plusieurs scénarios de consommation d'eau de boisson contaminée seront décrits dans cette étude.
\end{abstract}

ABSTRACT Dose coefficients from incorporated 129 Iodine. Influence of Dietary intake.

Internal dose coefficients recently published by the International Commission on Radiological Protection, and adopted in the new European Directive, allow direct calculation of the committed effective dose by multiplying the intake of radionuclide, expressed as becquerels, Bq, by those dose coefficients. For iodine, however, this methodology needs adaptation as iodine uptake by thyroid from blood is ruled by an adjustment process to the daily iodine intake: The greater that intake, the smaller the iodine fraction of thyroid uptake. Dealing with the 129 iodine radioactive isotope, for a same ingested $\mathrm{Bq}$, the uptake factor can vary, from 0.3 , for a daily dietary intake of $165 \mu \mathrm{g}$, to 0.03 for a daily dietary intake of $2.2 \mathrm{mg}$. Ilustrations of this variability are simulated in several situations that could involve contaminated water consumption.

IPSN/DPHD/SDOS BP6 92265 Fontenay-aux-Roses

** Hôpital Pitié Salpetrière, Service de Médecine Nucléaire, 47 Bd de l’Hôpital 75651 Paris Cédex 13

*** IPSN BP6 92265 Fontenay-aux-Roses 


\section{Introduction}

Au cours de leur fonctionnement, les réacteurs nucléaires produisent une quantité importante de produits de fission. À titre d'exemple, un réacteur à eau pressurisée de 900 MégaWatts (MW), produit environ $35 \mathrm{~kg} \mathrm{a}^{-1}$ de produits de fission à durée de vie inférieure à 30 ans et $50 \mathrm{~kg} \mathrm{a}^{-1}$ à durée de vie supérieure à 30 ans (Schapira, 1997). Parmi ces produits de fission à vie longue figure l'iode 129 , de période radioactive $1,57 \times 10^{7}$ ans; il est présent pour environ $80 \%$ de la totalité de l'iode produit. Si on envisage l'entreposage dans un site géologique profond de déchets radioactifs, l'hypothèse la plus pessimiste est le creusement d'un puits sur un site oublié après plusieurs siècles, et l'ingestion par les habitants de cette eau contaminée par l'iode 129 (European Commission, 1996). Dans ce cas, la consommation d'eau serait la voie d'atteinte prépondérante de l'homme.

Le retour à l'homme se fait soit à partir des effluents du retraitement dont les conséquences dosimétriques des rejets autorisés ont déjà été évaluées par ailleurs (Tubiana, 1982), soit à partir d'un stockage profond. Dans tous les cas, l'étude de l'impact dosimétrique de ${ }^{129}$ I rejeté dans l'environnement doit tenir compte de la présence d'iode stable présent dans la ration alimentaire ou dans l'air inhalé. En effet, les facteurs de dose par unité d'incorporation, recommandés par la Commission Internationale de Protection Radiologique (CIPR) sont calculés à l'aide de paramètres moyens de consommation journalière d'iode ne couvrant pas toutes les situations rencontrées lors des différents scénarios imaginés. On sait que la quantité d'iode présente dans la ration alimentaire influence le facteur de dose lié à l'incorporation d'iode radioactif (Book, 1977). Dans cette étude, nous supposerons que l'apport additionnel en iode est fourni seulement par l'eau de boisson contaminée par l'iode 129 lors de la rupture du confinement d'un stockage souterrain de combustible non retraité. Ces scénarios impliquent l'hypothèse pessimiste que l'iode reste dans le même état de solubilité dans les milieux naturels, sans réagir avec son environnement jusqu'à l'eau de boisson. Néanmoins, les résultats obtenus peuvent s'appliquer à toutes les situations où l'iode se retrouverait dans l'alimentation solide ou liquide ou dans l'air inhalé.

\section{Rappel du métabolisme de l'iode et de la physiologie de la thyroïde}

\subsection{Cycle de l'iode dans l'organisme}

L'iode, apporté par l'alimentation, est rapidement absorbé ( $90 \%$ en 1 heure) au niveau de l'estomac et de l'intestin grêle sous forme d'ion iodure $I^{-}$et se retrouve dans le plasma sanguin. De même, l'iode inhalé est transféré rapidement en totalité au sang. L'ion iodure présent dans le plasma est ensuite soit capté par la thyrö̈de, 
soit éliminé directement dans les urines. La fonction thyroïdienne concentre l'iodure dans un rapport de 25 à 1 pour synthétiser les hormones thyroïdiennes. Le captage de l'ion iodure par les cellules thyroïdiennes (thyréocytes) est un processus actif, effectué contre un gradient électrochimique. Ce captage est un processus adaptatif : c'est à dire que la fraction captée s'ajuste à la concentration de l'iode plasmatique liée directement à l'apport en iode (le plus souvent alimentaire). Finalement, le taux de captage de l'iode par la thyroïde reflète rigoureusement l'apport journalier. Ce phénomène adaptatif jouera un rôle déterminant dans le calcul des doses radiologiques consécutives à l'incorporation d'iode radioactif. Pour un becquerel incorporé, la dose à la thyroïde sera donc dépendante de la quantité d'iode stable présente dans le plasma.

Un schéma du métabolisme de l'iode chez l'adulte est donné en figure 1.

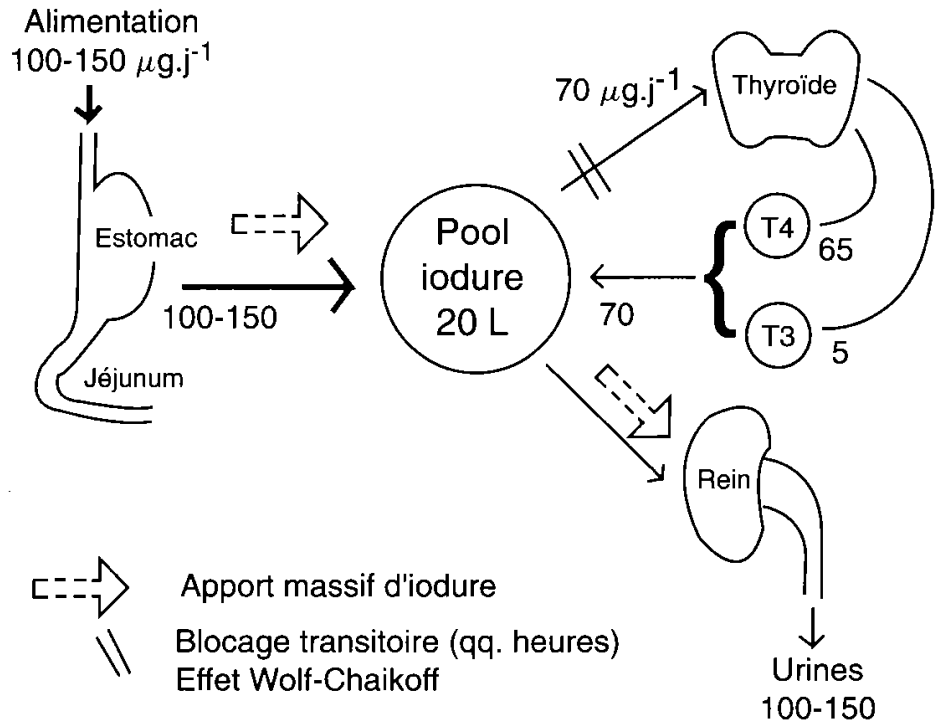

Fig. 1. - Modèle métabolique de l'iode (valeurs numériques pour un adulte). Iodine metabolic model (adult numerical values).

Dès son entrée dans la thyroïde, l'iodure est oxydé au sein des thyréocytes, puis incorporé à la thyroglobuline. C'est l'organification de l'iode qui passe de la forme minérale à la forme organique. Cette thyroglobuline est une protéine de poids moléculaire élevé, $M=660000$, synthétisée par la cellule thyroïdienne. Elle est stockée au sein de follicules thyrö̈diens dans un espace intercellulaire, la colloïde. Elle constitue une réserve d'hormones thyroïdiennes correspondant aux besoins de 30 à 60 jours. La thyroide contient, sous forme essentiellement de thyroglobuline, un stock d'environ 10 à $15 \mathrm{mg}$ d'iode. 
Selon les besoins, après retour dans les thyréocytes, la thyroglobuline subit une protéolyse. Elle libère, d'une part, 2 hormones thyrö̈diennes, la triiodothyronine (T3) et la tétraiodothyronine (T4 ou thyroxine) contenant respectivement 3 et 4 atomes d'iode par molécule, soit $59 \%$ et $65 \%$ de leur masse et, d'autre part, une fraction d'iode sous forme minérale pouvant participer à de nouvelles synthèses. Cette dégradation enzymatique de la thyroglobuline libère quotidiennement environ $10 \mu \mathrm{g}$ de $\mathrm{T} 3$ et $100 \mu \mathrm{g}$ de $\mathrm{T} 4$ dans le plasma (environ $70 \mu \mathrm{g}$ d'iode).

La thyroxine est une prohormone, qui est désiodée dans de nombreux tissus donnant la T3 de plus grande activité fonctionnelle. Cette désiodation est régulée selon les conditions métaboliques. Ainsi, pendant une période de jeûne, la désiodation diminue de manière significative et la thyroïde synthétise une proportion accrue de T3. Par conséquent, l'organisme dispose de multiples moyens pour faire face à une carence iodée.

Il existe dans l'organisme, un espace iodure en permanence alimenté par l'absorption digestive et par l'iode provenant de la désiodation de T3 et T4. L'iodure plasmatique est une partie du «pool iodure » extra-cellulaire (20 litres (1) contenant 50 à $100 \mu \mathrm{g}$ d'iodure). C'est dans cet espace iodure, représentant environ $30 \%$ du poids du corps, que puise constamment la thyroïde avec une

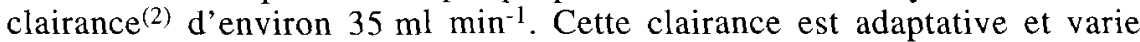
avec le facteur de captage. C'est aussi à partir de cet espace que s'effectue l'élimination urinaire. Celle-ci a une clairance de 40 à $50 \mathrm{ml} \mathrm{min}^{-1}$ non adaptative à l'inverse du captage thyroïdien. En situation d'équilibre, la quantité d'iode mesurée dans les urines recueillies pendant 24 heures est la meilleure estimation de l'ingestion quotidienne d'iode. La difficulté d'un recueil exhaustif, en particulier dans les enquêtes épidémiologiques, conduit souvent à estimer l'apport iodé quotidien par le rapport iodurie/créatininurie réalisable sur un simple échantillon urinaire. La créatininurie par 24 heures variant peu entre deux sujets du même âge, ce rapport est fortement corrélé à l'iodurie par $24 \mathrm{~h}$.

\subsection{Besoins physiologiques}

L'iode se retrouve un peu partout dans la nature et donc dans l'alimentation comme le montre le tableau I (Mornex, 1991).

(1) L (majuscule) est employé comme abréviation de titre pour éviter toutes confusions avec l'iode I.

(2) La clairance est un processus exponentiel décroissant qui est exprimé par un volume virtuel de plasma complètement épuré en 1 minute d'une substance par un organe (thyroïde ou rein). 
TABLEAU I

Iode dans la nature : valeurs moyennes (d'après R. Mornex, 1991). Iodine in the environment: mean values (from R. Mornex, 1991).

\begin{tabular}{|lr|}
\hline Eau de rivière & $<5 \mu \mathrm{g} \mathrm{L}^{-1}$ \\
Eau de pluie intérieure & $0,5 \mu \mathrm{g} \mathrm{L}^{-1}$ \\
Eau de pluie côtière & $5-10 \mu \mathrm{g} \mathrm{L}^{-1}$ \\
Eau de l'océan & $50 \mu \mathrm{g} \mathrm{L}^{-1}$ \\
Air marin & $10 \mu \mathrm{g} \mathrm{m}^{-3}$ \\
Air continental & $0,2 \mu \mathrm{g} \mathrm{m}^{-3}$ \\
Roches volcaniques & $500 \mu \mathrm{g} \mathrm{kg}^{-1}$ \\
Roches sédimentaires & $1500 \mu \mathrm{g} \mathrm{kg}^{-1}$ \\
\hline
\end{tabular}

On admet qu'un apport iodé quotidien de l'ordre de 125 à $150 \mu \mathrm{g}$ assure la couverture des besoins d'un sujet adulte. Les besoins en iode varient selon l'âge, le sexe et, chez la femme, pendant la grossesse ou l'allaitement (Tab. II) (Mornex, 1991).

\section{TABLEAU II}

Besoin quotidien en iode $\left(\mu \mathrm{g} \mathrm{j}^{-1}\right)$.

Iodine daily requirements $\left(\mu \mathrm{g} \mathrm{d}^{-1}\right)$.

\begin{tabular}{|cc|}
\hline Lourrisson & Iode $\boldsymbol{\mu \mathrm { g } \mathrm { j }} \mathbf{j}^{\mathbf{1}}$ \\
Enfant & $25-45$ \\
Homme & $50-100$ \\
Femme & 125 \\
Grossesse & $100-120$ \\
Allaitement & $125-200$ \\
\hline
\end{tabular}

Chez l'enfant en croissance ou lors de la puberté, l'importance de l'élimination rénale rend souhaitable l'apport d'une ration iodée supplémentaire de l'ordre de 150 à $200 \mu \mathrm{g}$ pour compenser le taux de renouvellement plus rapide que chez l'adulte. Des besoins analogues s'observent chez la femme enceinte. 


\subsection{Apport alimentaire}

Les sources naturelles de l'iode sont essentiellement alimentaires. Les apports iodés de l'alimentation sont d'origines variées comme le montre le tableau III. Les sources de provenance terrestre pures fournissent environ $50 \mu \mathrm{g} \mathrm{j}^{-1}$ (Mornex, 1991).

TABLEAU III

Sources d'iode : valeurs arrondies pour des produits non déshydratés en $\mu \mathrm{g} \mathrm{kg}^{-1}$ (d'après R. Mornex, 1991).

Iodine contents: rounded values for non deshydrated products in $\mu \mathrm{g} \mathbf{~ g g}^{-1}$ (from R. Mornex, 1991).

\begin{tabular}{|cccc|}
\hline Fruits & 20 & Poisson de mer & 250 \\
Poísson de rivière & 25 & Huîtres & 500 \\
Légumes & 30 & Langouste & 1000 \\
Céféales & $20-50$ & Morue & 1500 \\
Viande & 50 & Hareng & 3000 \\
Lait & 50 & Algues japonaises & $1000-4500$ \\
\hline
\end{tabular}

Les apports quotidiens moyens d'iode (estimés en $\mu \mathrm{g} \mathrm{j}^{-1} \mathrm{~d}^{\prime}$ iode urinaire ou

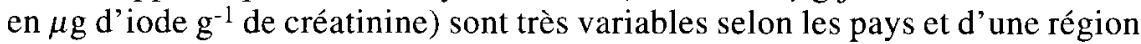
à l'autre de la France. Ces différences se retrouvent dans les mesures de l'iodurie qui varie selon les régions étudiées de $55 \mathrm{mg} \mathrm{g}^{-1}$ de créatinine dans la région Alsace à 174 dans la région Nord-Pas de Calais en passant par 86 dans l'est de la région Ile de France. Ces valeurs moyennes reflètent les habitudes alimentaires. Elles peuvent être modifiées par la prise de médicaments iodés tel la Cordarone ${ }^{\circledR}$ qui apporte par comprimé $6 \mathrm{mg}$ d'iodure. Il existe également des variations interindividuelles importantes.

Pour éviter les aléas de l'apport alimentaire, la plupart des nations ont procédé à un apport d'iode en iodant artificiellement le sel depuis 1920. En France, la législation permet depuis 1945 d'adjoindre jusqu'à $15 \mathrm{mg} \mathrm{kg}^{-1}$ de sel. Si l'on admet que les besoins en chlorure de sodium sont de l'ordre de $10 \mathrm{~g} \mathrm{j}^{-1}$, ceci devrait apporter une couverture iodée suffisante. Il faut cependant noter que l'iode présent dans l'eau de cuisson disparaît en grande partie pendant celle-ci.

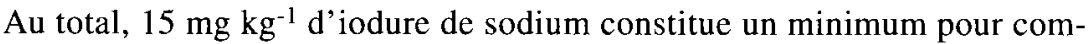
pléter l'apport naturel de l'alimentation. L'iodure de sodium contient $85 \%$ en masse d'iode, $15 \mathrm{mg}$ de $\mathrm{NaI}$ correspondent donc à $12,7 \mathrm{mg}$ d'iode. 


\subsection{Taux de captage journalier de l'iode par la thyroüde}

Le taux de captage est défini par la proportion d'atomes d'iode ingérés chaque jour se retrouvant dans la thyroïde au cours des 24 heures suivant l'incorporation. Le taux de captage journalier de l'iode par la thyroïde est fonction de l'apport alimentaire journalier.

Le corps thyroïde capte sans distinction l'iodure stable ou radioactif. Si l'on administre quotidiennement une quantité fixe d'iode radioactif, la concentration finale de la radioactivité dans la thyroïde sera très différente selon que le sujet dispose d'un espace iodure de $50 \mu \mathrm{g}$ ou de $500 \mu \mathrm{g}$. Il existe une corrélation directe entre ces valeurs (iode radioactif / iode total), c'est le processus de dilution isotopique.

La CIPR, qui indique, par ailleurs pour l'adulte de référence, un apport alimentaire moyen de $200 \mu \mathrm{g} \mathrm{j}^{-1}$ (CIPR 23, 1975), a recommandé un taux moyen de captage égal à 0,3 (ICRP 56, 1989).

Une étude dosimétrique précise exige que l'on tienne compte de l'apport alimentaire réel en iode et du taux de captage correspondant pour adapter convenablement les facteurs de doses à la situation considérée. Zvonova (1989) a donné la formule suivante pour calculer le taux de captage thyroïdien en fonction de l'apport journalier à partir de l'équilibre fonctionnel du modèle de Riggs (1952) :

$$
\mathrm{U}_{24}=70 /(70+\text { Inc }),
$$

où Inc est l'incorporation journalière d'iode exprimée en $\mu \mathrm{g}$.

La valeur $70(\mu \mathrm{g})$ est la quantité moyenne d'iode hormonal sécrétée quotidiennement. Elle est évaluée à partir de la compilation de plusieurs études faites chez différentes populations du globe.

La figure 2 montre, selon cette méthode, l'évolution du taux de captage en fonction de l'apport iodé quotidien.

En France, on estime que l'apport moyen d'iode dans l'alimentation varie de 85 à $100 \mu \mathrm{g}$. Pour un apport de $100 \mu \mathrm{g}$, le taux de captage de l'iodure plasmatique par la thyroïde est alors selon cette formule de 0,41 . On remarque que pour un apport quotidien deux fois plus grand $\left(200 \mathrm{mg} \mathrm{j}^{-1}\right), \mathrm{U}_{24}$ est égal à 0,26 et qu'il faut incorporer $2,6 \mathrm{mg} \mathrm{j}^{-1} \mathrm{~d}^{\prime}$ iode pour que le taux de captage chute d'un facteur 10 , et $26,8 \mathrm{mg} \mathrm{j}^{-1}$ pour qu'il chute d'un facteur 100 . Á l'inverse, dans une situation de carence dramatique, telle qu'on l'a observée parmi les populations de Nouvelle Guinée, le taux de captage peut atteindre 0,9 (Adams et al., 1968). 


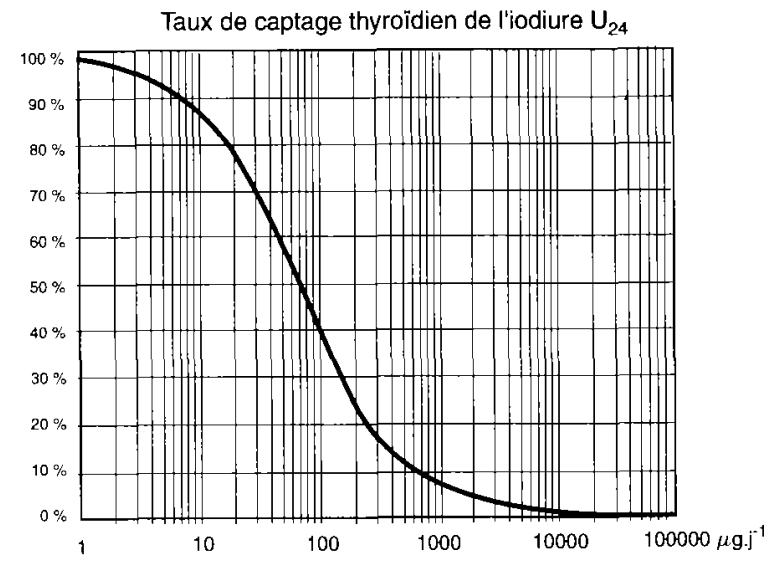

Fig. 2. - Taux de captage de l'iodure plasmatique par la thyrö̈de en fonction de l'apport journalier d'iode ( $\mu \mathrm{g})$.

Thyroid plasmatic iodide uptake fraction as a function of daily iodine intake ( $\mu \mathrm{g})$.

En l'absence de données spécifiques chez l'enfant, on suppose que l'âge n'influe pas sur le taux de captage de l'iode par la thyroïde. En revanche, le poids d'une thyroïde diffère entre un nourrisson et un adulte (de 1-2 g à 20-30 g). Par conséquent, chez l'enfant, la même activité est distribuée dans une masse de tissu plus petite ce qui conduit à une concentration plus élevée.

\subsection{Surcharge iodée brutale}

En cas de surcharge iodée brutale et massive, se produit un blocage transitoire:

- du captage de l'iodure par la thyroïde;

- de l'organification de l'iodure;

- de la libération des hormones T3 et T4 déjà formées.

Ce processus est appelé effet Wolff-Chaikoff (1948).

En cas de contamination accidentelle par l'iode radioactif, l'administration d'iode stable à forte dose en comprimés de $130 \mathrm{mg}$ de KI soit $100 \mathrm{mg}$ d'iode (Bhattacharyya et al., 1995) permet ainsi une diminution considérable de l'irradiation thyroïdienne à condition que cette prévention soit la plus précoce possible. L'effet est maximal si l'iode stable est pris quelques heures avant la contamination; il est 10 fois plus faible si la prise est faite 3 heures après et pratiquement nul si le délai dépasse 12 heures. La protection a une durée de 24 heures. La prise doit être renouvelée tous les jours tant que dure le risque de contamination. La dose d'iode stable à administrer dépend de l'âge (Rubery et al., 1990) (Tab. IV).

Normalement, la thyroïde s'adapte à la surcharge iodée et reprend rapidement une fonction normale avec un captage très réduit. On peut très rarement observer des 
hypothyroïdies (persistance du blocage tant que dure la surcharge avec guérison spontanée), des hyperthyroïdies transitoires ou des récidives d'hyperthyroïdies. Ces effets secondaires posent le problème de l'opportunité de la prévention par l'iode stable chez les sujets âgés et chez les adultes ayant des antécédents d'hyperthyroïdie.

\section{TABLEAU IV}

Masses d'iodure et d'iode nécessaires à la protection de la thyroïde par surcharge iodée selon l'âge.

Age dependent masses of iodide and iodine necessary to thyroid protection by iodine overload.

\begin{tabular}{|ccc|}
\hline Age & KI (mg) & lode (ng) \\
$0-1$ mois & 15 & 12,5 \\
1 mois -2 ans & 30 & 25 \\
3 ans -12 ans & 65 & 50 \\
$\quad>13$ ans & 130 & 100 \\
adulte, femme enceinte & & \\
\hline
\end{tabular}

\section{Méthodologie dosimétrique pour l'iode 129 ingéré}

\subsection{Données biocinétiques et dosimétriques de la CIPR}

La CIPR a utilisé un modèle recyclant simplifié à trois compartiments (Riggs, 1952) pour décrire le comportement biocinétique de l'iode dans l'organisme (Fig. 3).

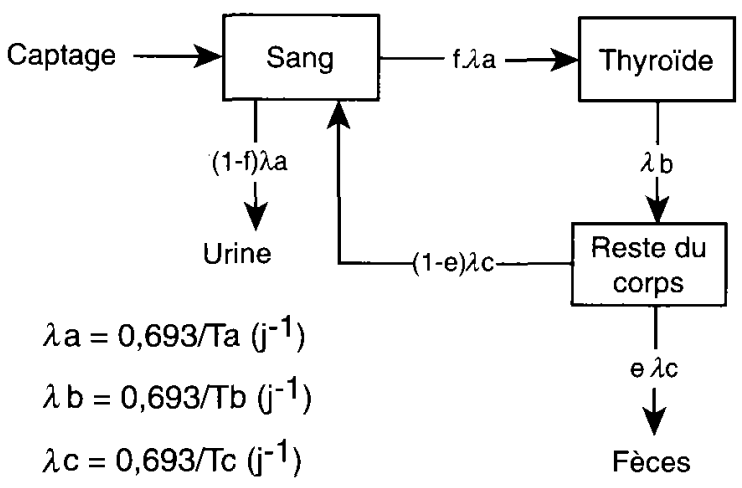

Fig. 3. - Modèle dosimétrique pour l'iode de la publication 56 de la CIPR (1989). Iodine dosimetric model from ICPR Publication 56 (1989). 
Le tableau $\mathrm{V}$ indique les valeurs des différents paramètres retenus par la CIPR intervenant selon l'âge, dans la biocinétique de l'iode, en particulier :

- $f_{1}$ fraction de l'activité ingérée absorbée par voie digestive et transférée au sang. L'absorption digestive se fait au niveau de l'intestin grêle pour tous les éléments dont la fraction $f_{1}$ est inférieure à 1 . Pour un $f_{1}$ de 1 , celle-ci s'effectue totalement au niveau de l'estomac sans passer dans les autres régions du tractus gastrointestinal (CIPR, 1979);

- périodes biologiques correspondant aux coefficients de transfert entre chacun des compartiments ;

- périodes biologiques apparentes de l'iode thyroïdien résultant du recyclage de celui-ci.

\section{TABLEAU $V$}

Valeurs des paramètres du modèle biocinétique de l'iode en fonction de l'âge (CIPR Publication 67, 1993).

Age dependent biokinetic parameters values for the iodine model (ICRP Publication 67, 1993).

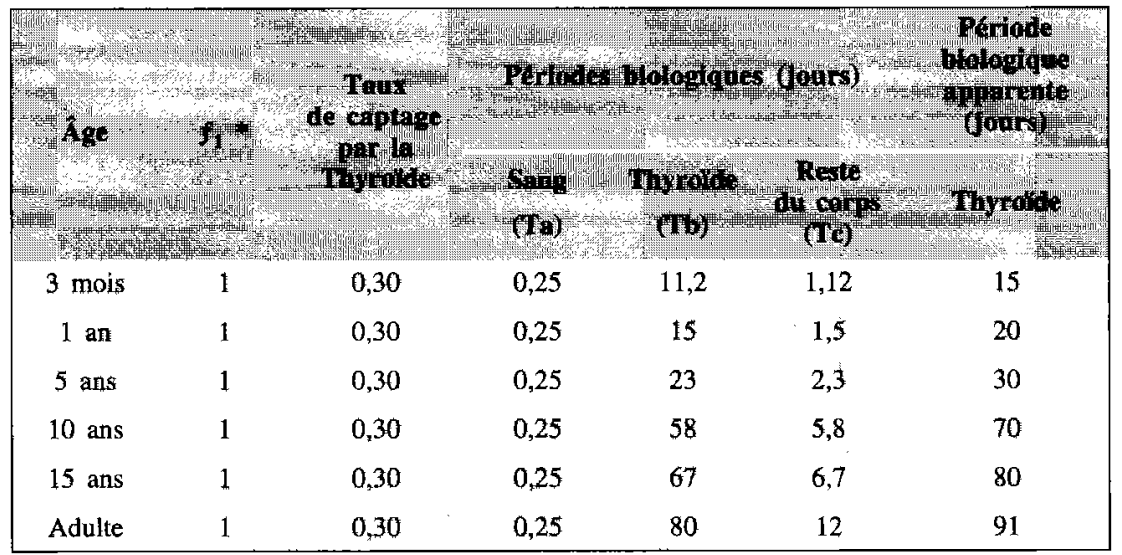

\subsection{Doses engagées par unité d'incorporation (DPUI) par ingestion}

La publication 67 de la CIPR (1993) fournit les coefficients de doses par ingestion, pour l'adulte et les enfants, en utilisant un taux de captage thyroïdien égal à 0,3 . La dose délivrée à la thyroïde contribue à la dose efficace dans la proportion de $95 \%$. Chez l'adulte, la dose équivalente à la thyroïde et la dose efficace sont engagées sur 50 ans. Chez l'enfant, les coefficients de doses engagées sont calculés jusqu'à l'âge de 70 ans. Les classes d'âge recouvrent par convention les périodes suivantes pour 3 mois (de 0 à 1 an), 1 an (de 1 an 
à 2 ans), 5 ans (de 2 ans à 7 ans), 10 ans (de 7 ans à 12 ans), 15 ans (de 12 ans à 17 ans) et pour l'adulte (au-delà de 17 ans) (CIPR, 1989).

Par comparaison avec l'adulte, ces coefficients ne varient chez l'enfant que d'un facteur 2 au maximum. Ceci résulte du fait que pour une même quantité incorporée, la concentration est plus élevée dans l'organe, mais l'iode étant éliminé plus rapidement, ces deux paramètres se compensent globalement.

De plus, la quantité ingérée est plus faible chez l'enfant qui consomme moins d'eau que l'adulte (CIPR, 1975). Le tableau VI indique, en fonction de l'âge, les valeurs des doses équivalentes et efficaces ainsi obtenues et les valeurs standard des volumes ingérés. Ces facteurs dosimétriques sont établis quel que soit l'âge avec un taux de captage de 0,3 .

\section{TABLEAU VI}

Paramètres de référence pris en compte pour les calculs dosimétriques; DPUIs ingestion en fonction de l'âge (CIPR, 1993) et quantités d'eau ingérées par l'homme de référence (CIPR, 1975).

Reference parameters for dose calculation; Age dependent ingestion DPUI (ICRP, 1993) and ingested water quantities by the reference man (ICRP, 1975).

\begin{tabular}{|c|c|c|c|c|c|c|}
\hline Age a 11.pogforption & 3 mois & $1 \mathrm{an}$ & 5 ans & 10 ans & 15 ans & Adalte \\
\hline $\begin{array}{c}\text { Dose equivalente à la } \\
\text { thyroïde, } H_{\text {th }} \\
\left(\mathrm{Sv} \mathrm{Bq}^{-1}\right)\end{array}$ & $3,7 \times$ & $4,3 \times 10^{-6}$ & 3,4 & $3,8 \times 10^{-6}$ & 2,8 & 2,1 \\
\hline $\begin{array}{l}\text { Dose efficace, } E \\
\quad\left(\text { Sv } \mathbf{B q}^{-1}\right)\end{array}$ & $1,8 \times 10^{-7}$ & $2,2 \times 10^{-7}$ & $1,7 \times 10^{-7}$ & $1,9 \times 10^{-7}$ & $1,4 \times 10^{-7}$ & $1,1 \times 10^{-7}$ \\
\hline Eau ingérée $\left(\mathrm{L} \mathrm{j}^{-1}\right)$ & 1 & 1 & 1,25 & 1,5 & 1,75 & 2 \\
\hline
\end{tabular}

Notons que, pour l'inhalation d'iode, le transfert au sang est instantané pour la forme gazeuse, et très rapide (Type F) pour les aérosols. Les DPUIs pour les gaz sont sensiblement les mêmes que pour l'ingestion, elles sont un peu plus faibles pour les aérosols (CIPR, 1995).

\subsection{Doses engagées Par Unité d'Incorporation selon le taux de captage}

La dose délivrée à la thyroïde est proportionnelle à la quantité d'iode incorporée par celle-ci et inversement proportionnelle à la masse de la thyroïde qui intervient dans le calcul de l'énergie massique efficace. Par la suite, pour les calculs de doses, nous considérerons qu'une modification du régime alimentaire iodée n'entraîne pas de changement de la masse thyroïdienne. La quantité d'iode incorporée dans la thyroïde est liée au taux de captage qui fixe la proportion d'iode transférée à la thyroïde. Les doses délivrées sont alors proportionnelles 
au taux de captage. Il est alors indispensable d'extrapoler les DPUIs et de les adapter aux différentes valeurs de l'ingestion journalière en calculant la valeur du facteur de captage journalier $\left(\mathrm{U}_{24}\right)$ et en l'utilisant dans la relation simple :

$$
\text { DPUI }=\text { DPUI }_{67} \times \mathrm{U}_{24} / 0,30 \text {. }
$$

DPUI $_{67}$ étant la valeur du coefficient de dose établi par la publication 67 de la CIPR et reprise dans la publication 72 , et valeur de référence pour tous les paramètres pris en compte dans le calcul de la dose délivrée aux organes : énergie des rayonnements, géométrie, irradiation réciproque des organes, etc.

Enfin la valeur de l'activité spécifique de l'iode 129 varie légèrement dans la littérature. Nous avons adopté, pour les calculs, les valeurs suivantes : activité spécifique égale à $6,53 \mathrm{~Bq} \mu \mathrm{g}^{-1}$ d'iode 129 correspondant à une période radioactive de $1,57 \times 10^{7}$ ans donnée par la CIPR dans la publication 38 (1983).

\section{Application à des scénarios d'incorporation par une eau de boisson contaminée}

\subsection{Les scénarios étudiés}

Les exemples suivants ont été choisis pour illustrer l'application de la méthodologie dosimétrique pour des valeurs soit extrêmes et démonstratives soit plus proches d'une éventuelle fuite d'un stockage.

Nous avons adopté la valeur de $100 \mu \mathrm{g} \mathrm{j}^{-1}$ (Mornex, 1991) comme quantité moyenne d'iode ingérée dans l'alimentation par un adulte en France. Pour cette valeur, le taux de captage par la thyroide est de 0,41 . Ces chiffres sont également retenus pour les enfants en l'absence de données spécifiques.

Deux types de scénarios sont étudiés dans ce document:

Dans le premier type, on considère trois cas de contamination de l'eau de boisson, par un mélange d'iode composé de $80 \%{ }^{129} \mathrm{I}+20 \%{ }^{127} \mathrm{I}$ qui viendrait s'ajouter à la ration d'iode alimentaire non radioactif.

- cas $\mathrm{n}^{\circ} 1$ : $35 \mathrm{mg} \mathrm{a}^{-1}$, correspondant à $100 \mu \mathrm{g}$ par jour du mélange : soit $80 \mu \mathrm{g}$ de ${ }^{129} \mathrm{I}$ ou environ $520 \mathrm{~Bq} \mathrm{j}^{-1}$ (équivalent à $2 \mathrm{~L}$ d'eau par jour à la concentration de $50 \mu \mathrm{g} \mathrm{\textrm {L } ^ { - 1 }}$ de ce mélange).

- cas $\mathbf{n}^{\circ} 2$ : $3,5 \mathrm{mg} \mathrm{a}^{-1}$, correspondant à $10 \mu \mathrm{g}$ par jour du mélange : soit $8 \mu \mathrm{g}$ de ${ }^{129} \mathrm{I}$ ou environ $52 \mathrm{~Bq} \mathrm{j}^{-1}$ (équivalent à $2 \mathrm{~L}$ d'eau par jour à la concentration de $5 \mu \mathrm{g} \mathrm{L}^{-1}$ de ce mélange).

- cas $\mathrm{n}^{\circ} \mathbf{3}: 0,35 \mathrm{mg} \mathrm{a}^{-1}$, correspondant à $1 \mu \mathrm{g}$ par jour du mélange : soit $0,8 \mu \mathrm{g}$ de ${ }^{129} \mathrm{I}$ ou environ $5,2 \mathrm{~Bq} \mathrm{j}^{-1}$ (équivalent à $2 \mathrm{~L}$ d'eau par jour à la concentration de $0,5 \mu \mathrm{g} \mathrm{L^{-1 }}$ de ce mélange). 
Dans le second type, on considère un cas concernant un stockage perdant 0,1 mole d'iode 129 soit 12,9 g par an (tout l'iode labile des déchets étant instantanément libéré).

- cas $\mathrm{n}^{\circ} 4$ : Tout l'iode est déversé dans un puits au débit de $3,10^{5} \mathrm{~m}^{3}$ par an. La concentration est de $4,3 \times 10^{-8} \mathrm{~g}^{129} \mathrm{I} \mathrm{L}^{-1}$, et, pour l'absorption de $2 \mathrm{~L} \mathrm{j}^{-1}$, l'activité ingérée est de $0,56 \mathrm{~Bq} \mathrm{j}^{-1}$.

Le tableau VII montre, pour l'adulte français ayant un apport iodé moyen journalier arrondi à $100 \mu \mathrm{g}$, les valeurs de $\mathrm{U}_{24}$ découlant d'une quantité ajoutée $Q_{\mathrm{j}}$ d'iode 129 et les facteurs de doses correspondant à ces scénarios. Les taux de captage varie pour les cas $n^{\circ} 1$ et $n^{\circ} 2$ parce que la quantité d'iode ajoutée à la ration journalière $\left(Q_{j}\right)$ est importante; en revanche, pour les cas $n^{\circ} 3$ et 4 , le taux de captage reste inchangé puisque l'iode 129 est soit une fraction du régime alimentaire (modification du rapport isotopique) soit en quantité trop faible pour influencer $\mathrm{U}_{24}$.

La figure 4 montre la variation de la DPUI en fonction de l'apport d'iode 129 . Pour la France, celle-ci reste sensiblement stable jusqu'à une valeur de $\mu \mathrm{g} \mathrm{j}^{-1}$ et diminue pour des valeurs supérieures et atteint des valeurs très faibles pour des quantités très grandes d'iode ingérées quotidiennement (à partir de $15 \mathrm{mg} \mathrm{j}^{-1}$ ) que l'on rencontre uniquement dans des situations particulières, tels les traitements médicaux.

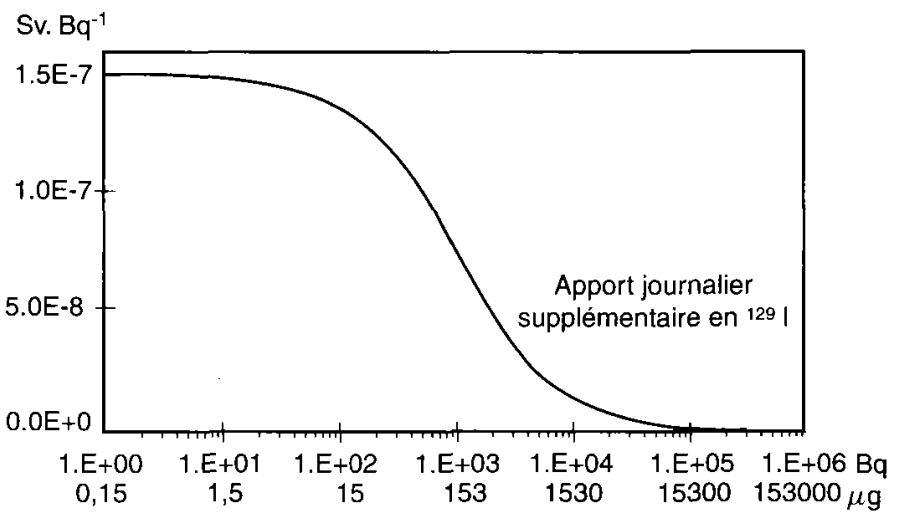

Fig. 4. - Évolution de la DPUI efficace engagée en fonction de l'apport supplémentaire quotidien en iode 129 pour la France.

Evolution of the committed effective DPUI as a function of 129 iodine quantity added over the daily amount in France. 


\section{TABLEAU VII}

DPUIs équivalentes et efficaces pour un sujet adulte en fonction de la quantité $Q_{\mathrm{j}}$ d'iode 129 ajoutée au régime alimentaire.

Adult equivalent and effective DPUI as a function of $Q_{\mathrm{j}}$, 129 iodine quantity added over the dietary amount.

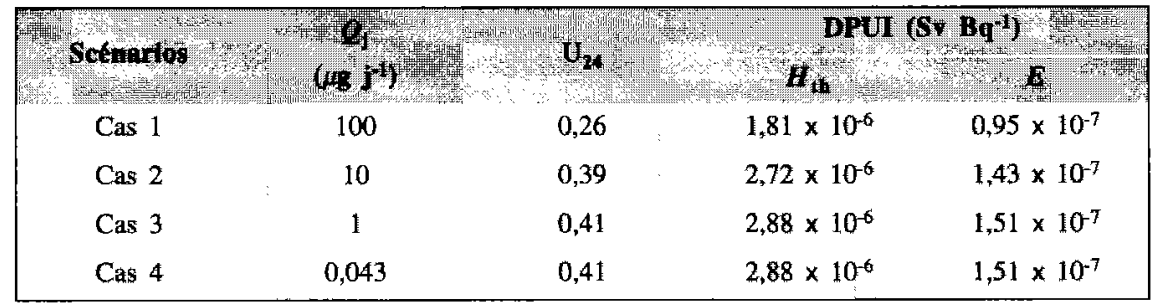

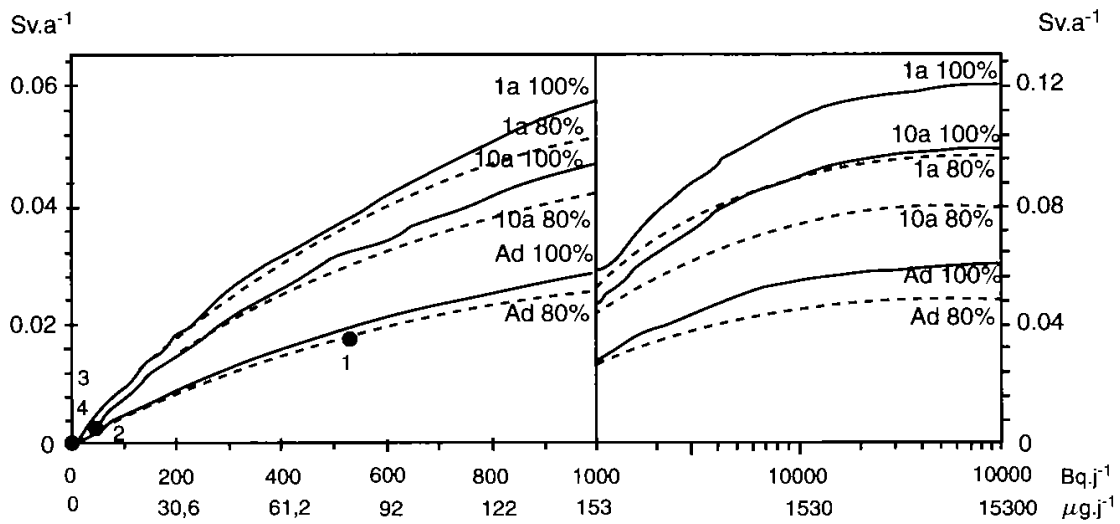

Fig. 5. - Évolution de la dose efficace engagée en fonction de l'apport journalier supplémentaire en iode au régime alimentaire français, par l'ingestion d'iode 129 soit pur (100\%) soit à $80 \%$ d'iode 129, chez l'adulte (Ad) et les enfants de 1 an (Ia) et de 10 ans (10a). Les points représentent la position des cinq cas analysés pour l'adulte.

Evolution of the committed effective dose as a function of the daily iodine intake added over the french dietary amount, from 129 iodine ingestion either pure (100\%) either $80 \%$ of 129 iodine, in adults (Ad), 1 year-olds (1a) and 10 year-olds (10a). Dots indicate the five adult case positions.

\subsection{Doses délivrées par l'iode 129 dans l'eau de boisson ingérée en France}

Les calculs sont faits pour l'adulte, et les enfants de 1 et 10 ans, français. Les résultats dosimétriques sont donnés pour une contamination quotidienne d'une durée d'un an (Tab. VIII). 


\section{TABLEAU VIII}

Doses délivrées à l'adulte et aux enfants après ingestion quotidienne d'eau contaminée à l'iode 129 pour les quatre cas décrits.

Doses to adult and children subjects from daily ingestion of 129 iodine contaminated water in the four described cases.

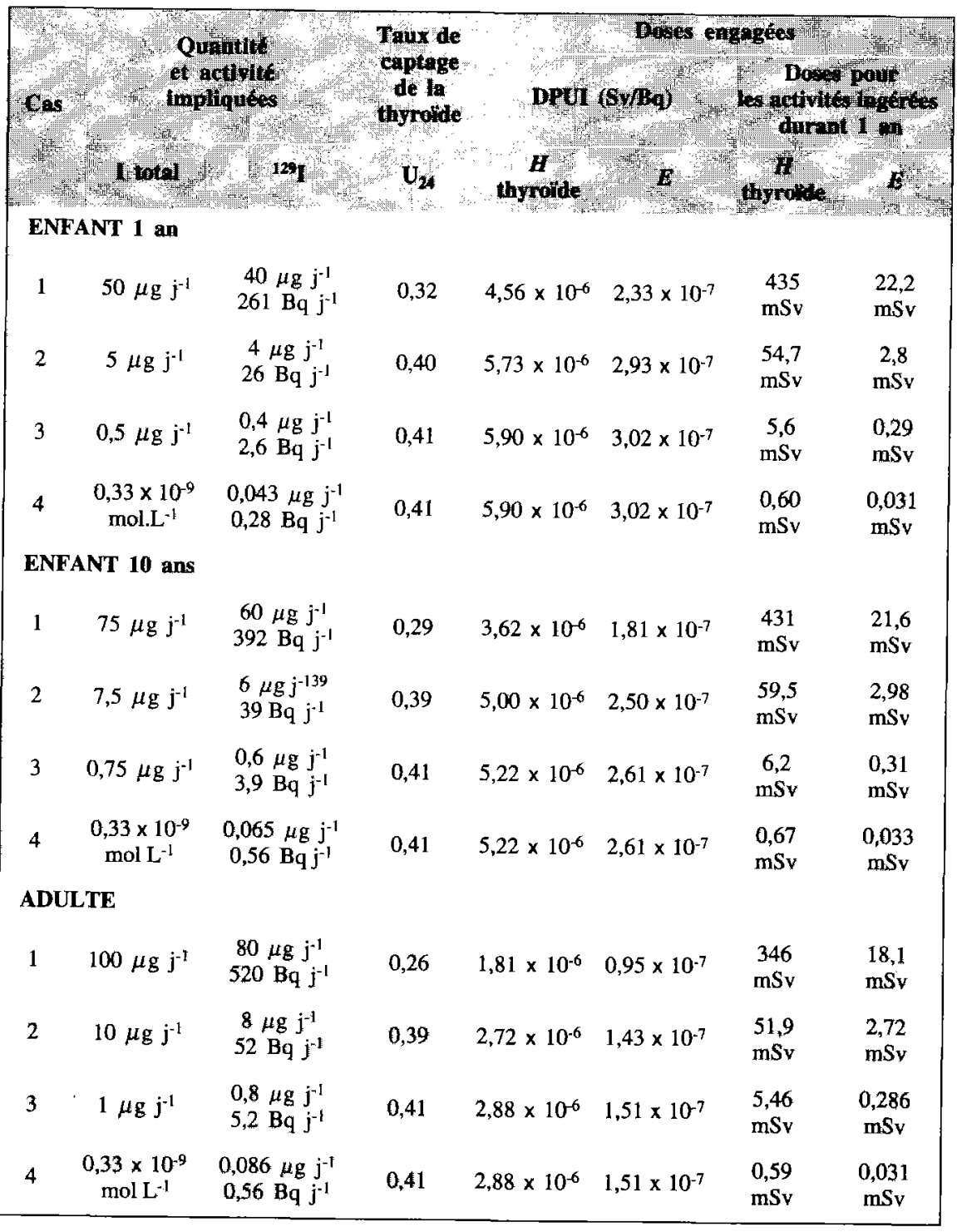


Les valeurs ainsi calculées sont reportées sur les courbes théoriques (Fig. 5) qui montrent l'évolution de la dose en fonction de l'apport journalier supplémentaire en iode 129 pur ou à $80 \%$. Pour rendre la figure plus lisible, en abscisse, la première partie de l'échelle est linéaire et la deuxième logarithmique.

\subsection{Doses délivrées par l'Iode 129 dans l'eau de boisson dans d'autres pays}

Le régime iodé est variable d'un pays à l'autre et présente des valeurs extrêmes (Tab. IX). En Allemagne et en Roumanie, 1'apport naturel est pauvre en iode. En France et aux USA, un apport iodé supplémentaire est obtenu par addition d'iode dans le sel consommé. Au Japon, l'alimentation est riche en jode de façon naturelle (consommation d'algues).

Les taux de captage qui en découlent pour ces différents pays, entraînent pour chacun, des coefficients de doses spécifiques. Pour un apport d'iode 129 en faible quantité pondérable ne modifiant pas $U_{24}$, la dose pour la population américaine est la même que celle de la CIPR. Celle de la population allemande est environ 2 fois plus forte et celle de la population japonaise environ 8 fois plus faible.

\section{TABLEAU IX}

Doses délivrées à l'adulte et aux enfants après ingestion quotidienne d'eau contaminée.

Doses to adult and children from daily ingestion of 129 iodine contaminated water.

\begin{tabular}{|c|c|c|c|c|c|}
\hline 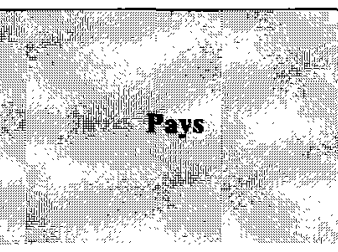 & $\begin{array}{l}\text { Apporit } \\
\text { alimen } \\
\text { tare iod } \\
\text { mgyen } \\
\text { (valeur } \\
\text { arrondite } \\
\text { ng it }\end{array}$ & 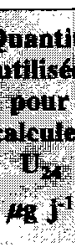 & 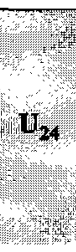 & 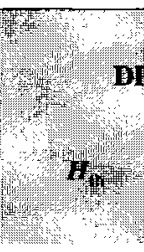 & 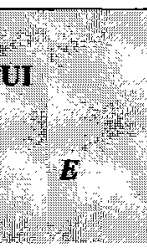 \\
\hline $\begin{array}{l}\text { Allemagne Centre Baltique } \\
\text { (Anke 1993) }\end{array}$ & $\begin{array}{l}50 \\
60\end{array}$ & 55 & 0,57 & $3,90 \times 10^{-6}$ & 2,05 \\
\hline Roumanie (Simescu 1993) & 68 & 68 & 0,50 & $3,55 \times 10^{-6}$ & $1,86 \times 10^{-7}$ \\
\hline France (Mornex 1991) & 100 & 100 & 0.41 & $2,88 \times 10^{6}$ & $1,51 \times 10^{7}$ \\
\hline USA (Pino 1993) & 165 & 165 & 0,30 & $2,10 \times 10^{6}$ & $1,10 \times 10^{-7}$ \\
\hline Japon (Nagataki 1993) & 1865 & 1865 & 0,036 & $2,50 \times 10^{-7}$ & $1,33 \times 10^{-8}$ \\
\hline
\end{tabular}


La figure 6 montre les variations importantes de la dose équivalente thyroïdienne par unité d'incorporation en fonction de l'apport journalier selon les pays considérés.

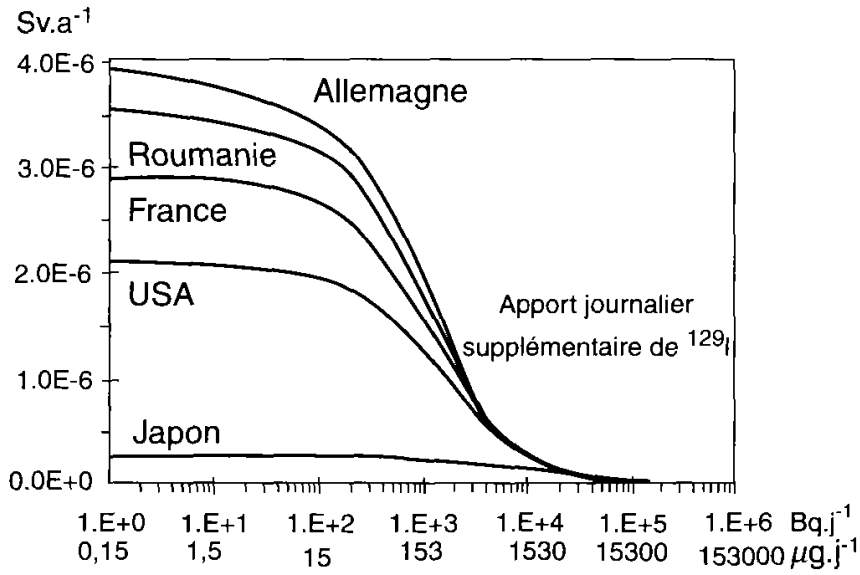

Fig. 6. - DPUI équivalente thyroüdienne engagée en fonction de l'apport journalier supplémentaire en iode 129 pur selon les pays.

Commited thyroüd equivalent DPUI as a function of 129 iodine intake added over the daily amount in different countries.

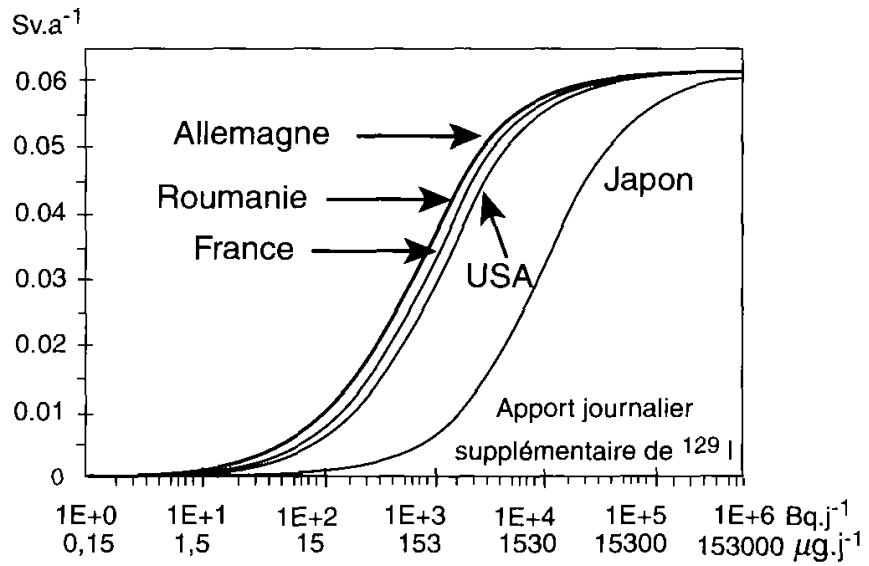

Fig. 7. - Dose efficace engagée pour la quantité ingérée en une année en fonction de l'apport journalier supplementaire en iode 129 pur selon les pays.

Committed effective dose from the quantity ingested over one year as a function of pure 129 iodine intake added over the daily amount in different countries. 
La figure 7 permet la comparaison des courbes des doses efficaces engagées correspondant à une consommation quotidienne d'iode 129 durant 1 an pour différents pays. Elles sont assez voisines pour l'Allemagne, la Roumanie, la France et les USA. En revanche, la courbe concernant le Japon s'en éloigne très nettement montrant l'influence de l'apport iodé de 10 à 30 fois plus élevé que celui des autres pays considérés, conduisant à un taux de captage faible $(0,036)$.

\section{Conclusion}

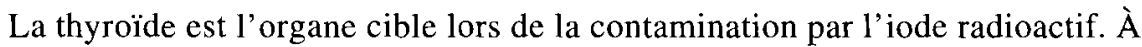
la suite d'une incorporation, la thyroïde exerce vis à vis de l'iode contenu dans le sang une clairance adaptative qui lui permet de ne prélever que la quantité d'iode nécessaire à ses besoins. C'est cette quantité qui entraîne l'exposition interne de cet organe. Elle peut constituer une fraction variable de l'activité selon que la quantité totale de l'iode disponible provenant de l'ingestion compense juste les besoins en iode de la thyroïde ou leur est supérieure. Elle se composerait dans les scénarios étudiés de l'iode stable alimentaire augmenté de l'apport de l'iode radioactif en provenance du stockage des déchets. La régulation peut être représentée par un facteur de captage quotidien de l'iode plasmatique intervenant de façon linéaire dans le calcul de la dose délivrée au tissu thyroïdien. On sait que l'activité spécifique de l'iode 129 contenu est égale à $6,53 \mathrm{~Bq}$ par $\mu \mathrm{g}$ d'iode, et l'on peut connaître avec assez de précision l'apport alimentaire en iode stable par lequel cette activité est diluée dans le sang. Dans un grand nombre de pays, dont la France, l'apport alimentaire est voisin de $100 \mu \mathrm{g}$ par jour et légèrement inférieur à la couverture des besoins d'un sujet adulte, ce qui entraîne un facteur de captage de 0,41 , supérieur à celui qui est indiqué dans le modèle métabolique de la CIPR. La DPUI à la thyroïde calculée avec ce facteur est donc légèrement supérieure à celle de la Publication 67 de la CIPR, $\left(2,88 \times 10^{-6} \mathrm{~Sv}\right.$ au lieu de $\left.2,10 \times 10^{-6} \mathrm{~Sv}\right)$ chez l'adulte.

\section{RÉFÉRENCES}

Adams D.D., Kennedy T.H., Choufoer J.C., Querido A. (1968) Endemic goitre in western New Guinea. III. Thyroid-stimulating activity of serum from severely iodine-deficient people. J. Clin. Endocrinol. Metab., 28, 685-692.

Anke M., Groppel B., Bauch, K.H. (1993) Iodine in the food chain. In : Iodine deficiency in Europe; A continuing concern; F. Delange, J. T. Dunn, D. Glinoer Eds. NATO ASI Series Life Sci., 241, 151-157.

Bhattacharyya M.H., Breitenstein B.D., Métivier H., Muggenburg B.A., Stradling G.N., Volf V. (1995) Traitement de la contamination interne accidentelle des travailleurs. Collection IPSN. 
Book S.A. (1977) Iodine 129 : Limits to radiologic dose, Health Phys. 32, 261-266.

CCE, Directive 96/29 EURATOM du conseil du 13 mai 1996, fixant les normes de base relatives à la protection sanitaire de la population et des travailleurs contre les dangers résultant des rayonnements ionisants. J.O.C.E. n ${ }^{\circ} 159$ du 29 juin 1996.

European Commission (1996) Evaluation of elements responsible for the effective dose rates associated with the final storage of radioactive waste: EVEREST project, summary report, EUR $17122 \mathrm{EN}$.

ICRP, International Commission On Radiological Protection (ICRP) (1975) Report of the task group on reference man, ICRP Publication 23, Oxford, Pergamon press.

ICRP, International Commission On Radiological Protection (ICRP) (1979) Limits for Intakes of Radionuclides by Workers, ICRP Publication 30, Oxford, Pergamon press.

ICRP, International Commission On Radiological Protection (ICRP) (1983) Radionuclide transformations, ICRP Publication 38, Oxford, Pergamon press.

ICRP, International Commission On Radiological Protection (ICRP) (1989) Age Dependent Doses to Members of the Public from Intake of Radionuclides, ICRP Publication 56 Part 1, Oxford, Pergamon press.

ICRP, International Commission On Radiological Protection (ICRP) (1993) Age Dependent Doses to Members of the Public from Intake of Radionuclides: Part 2 Ingestion dose coefficients, ICRP Publication 67, Oxford, Elsevier Science Ltd.

ICRP, International Commission On Radiological Protection (ICRP) (1995) Age Dependent Doses to Members of the Public from Intake of Radionuclides: Part 4 Inhalation dose coefficients, ICRP Publication 71 Oxford, Elsevier Science Ltd.

Mornex R. (1991) Métabolisme de l'iode et besoins alimentaires. Dans : Irradiation par l'iode radioactif. C. R. journée organisée par le Comité de Radioprotection EDF, Paris, 25 octobre 1991.

Nagataki S. (1993) Status of iodine nutrition in Japan: In: Iodine deficiency in Europe ; A continuing concern; F. Delange, J.T. Dunn, D. Glinoer Eds. NATO ASI Ser. Life Sci., 241, 141-146.

Pino S., Braveman L.E. (1993) Status of iodine nutrition in the United States. In : Iodine deficiency in Europe; A continuing concern; F. Delange, J.T. Dunn, D. Glinoer Eds. NATO ASI Ser. Life Sci., 241, 129-13.

Riggs D.S. (1952) Quantitative aspects of iodine metabolism in man, Pharmacol. Rev. 4, 284370 .

Rubery E., Smales E. (1990) Iodine prophylaxis following nuclear accidents, Oxford Pergamon Press, 1-182.

Schapira J.P. (1997) Les déchets nucléaires, un dossier scientifique (R. Turlay, Ed.). Les Éditions de Physique, Les Ulis.

Simescu M., Popescu R., Ionitiu D., Zbrana E., Grecu E., Marinescu E., Tintea L., Nicolaescu E., Purice M., Popa M., and Gutekunst R. (1993) The status of iodine nutrition in Romania. In : Iodine deficiency in Europe; A continuing concern (F. Delange, J.T. Dunn, D. Glinoer Eds) NATO ASI Ser. Life Sci. 241, 151-157.

Tubiana M. (1982) Métabolisme et Radiotoxicité de l'iode radioactif. Toxiques Nucléaires (P. Galle Ed) Masson, Paris, 53-77.

Wolff J., Chaikoff I.L. (1948) The inhibitory action of excessive iodine upon the synthesis of diiodotyrosine and of thyroxine in the thyroïd gland of the normal rat. Endocrinology 43, 174-179.

Zvonova I. (1989) Dietary intake of stable I and some aspects of radioiodine dosimetry. Health Phys. 57, 471-475. 